\title{
Numerical Approaches to Linear-Quadratic Differential Games with Imperfect Observations
}

\author{
by ARUNABHA BAGCHI $\dagger$
}

Department of System Science, School of Engineering and Applied Science, University of California, Los Angeles, U.S.A.

and GEERT JAN OLSDER

Department of Applied Mathematics, Twente University of Technology, Enschede, The Netherlands

ABSTRACT: A two-person pursuit-evasion stochastic differential game with state and measurements corrupted by noises is considered. In an earlier paper the problem was reformulated and solved in an infinite-dimensional-state space, and the existence of saddle-point solutions under certain conditions was proved. The present paper provides a numerical solution for the resulting continuous-time integro-partial differential equations. This solution scheme is based on the utilization of the second guessing technique, and, in spite of the fact that a complicated set of integro-partial differential equations have to be solved, the numerical results seem plausible and promising.

\section{Introduction}

We consider a two-person pursuit-evasion stochastic differential game in which the system state and the measurements are affected by (possibly independent) noises. The feature that the players have access to different noisy measurements makes this problem considerably harder than the traditional one where the players have access to complete, identical information. Wilman (1), in a seminal work, solved the discrete version of the problem and used complicated limiting operations to obtain the result for the continuous-time case. Direct attempts to solve this problem have recently attracted attention $(\mathbf{2}, \mathbf{3})$.

The present paper is a continuation of the results obtained in (2). In that paper, the existence of saddle-point solutions has been established under certain sufficient conditions for linear system dynamics and quadratic pay-off. An infinitedimensional state space was introduced in which the problem was reformulated and solved. The optimal (linear) strategies were characterized by a set of 34 coupled integro-partial differential equations.

The present paper reports on a numerical solution of these integro-partial

$\dagger$ On sabbatical leave from Twente University of Technology, Department of Applied Mathematics, P.O. Box 217, 7500 AE Enschede, The Netherlands. 
differential equations. Some numerical results were discussed in (1) for discrete-time game problems. We present numerical solutions here for the continuous-time equations.

The outline of the paper is as follows. In Section II, the mathematical problem statement is given. A method of solution is presented in Section III based on the idea of second-guessing technique. The numerical results presented here are, however, based upon the solution method given originally in (2) and explained briefly in Section IV. The results in (2) are slightly extended to include the case of a non-zero initial condition. In Section V, a numerical scheme for solving the problem is presented and actual numerical results for a scalar example are given in Section VI.

\section{Problem Statement}

We consider a direct two-player extension of the standard linear-quadratic Gaussian control problem as discussed in standard textbooks, (4). The differential equation describing the evolution of the system in state space is given by

$$
\begin{aligned}
& \dot{x}(t ; \omega)=A(t) x(t ; \omega)+B_{\mathrm{p}}(t) u_{\mathrm{p}}(t ; \omega)+B_{\mathrm{e}}(t) u_{\mathrm{e}}(t ; \omega)+F(t) n(t ; \omega) ; \quad 0 \leqslant t \leqslant T, \\
& x(0 ; \omega)=x_{0}
\end{aligned}
$$

where $x(t ; \omega)$ is an $n$-vector denoting the state; $u_{\mathrm{p}}(t ; \omega)$ is an $r_{\mathrm{p}}$-vector denoting the strategy of one player-the pursuer; $u_{\mathrm{e}}(t ; \omega)$ is an $r_{\mathrm{c}}$-vector denoting the strategy of the other player - the evader; $n(\cdot ; \omega)$ is a $q$-vector representing white noise with unit intensity and zero mean; and $T$ is the prescribed final time.

The matrices $A(t), B_{\mathrm{p}}(t), B_{\mathrm{e}}(t)$ and $F(t)$ have appropriate dimensions. The initial condition $x_{0}$ is assumed to be normally distributed with mean $\bar{x}_{0}$ and covariance matrix $P_{0}$. We use the $L_{2}$-theory of white noise developed by Balakrishnan (5), as opposed to the conventional Wiener process approach. For linear strategies, both the approaches lead to identical results, but the $L_{2}$-theory is much easier to work with in our present context as explained in (2).

The observations available to the players are

$$
\begin{gathered}
y_{\mathrm{p}}(t ; \omega)=C_{\mathrm{p}}(t) x(t ; \omega)+G_{\mathrm{p}}(t) n(t ; \omega), \text { pursuer }, \\
y_{\mathrm{e}}(t ; \omega)=C_{\mathrm{e}}(t) x(t ; \omega)+G_{\mathrm{e}}(t) n(t ; \omega), \text { evader, }
\end{gathered}
$$

where $y_{\mathrm{p}}$ and $y_{\mathrm{e}}$ are $m_{\mathrm{p}}$ - and $m_{\mathrm{e}}$-dimensional vectors, respectively. The matrices $C_{\mathrm{p}}$, $C_{\mathrm{e}}, G_{\mathrm{p}}, G_{\mathrm{e}}$ have appropriate dimensions.

The criterion that the pursuer wants to minimize and the evader wants to maximize is given by $\mathrm{E} J$ where

$$
\begin{aligned}
J=\frac{1}{2}\left\{\left\langlex(T ; \omega), Q_{\mathrm{f}} x(T\right.\right. & ; \omega)\rangle+\int_{0}^{T}[\langle x(t ; \omega), Q(t) x(t ; \omega)\rangle \\
& \left.\left.+\left\langle u_{\mathrm{p}}(t ; \omega), R_{\mathrm{p}}(t) u_{\mathrm{p}}(t ; \omega)\right\rangle-\left\langle u_{\mathrm{e}}(t ; \omega), R_{\mathrm{e}}(t) u_{\mathrm{c}}(t ; \omega)\right\rangle\right]\right\} \mathrm{d} t
\end{aligned}
$$

and E denotes "expectation". The admissible strategies $u_{\mathrm{p}}(t ; \omega)$ and $u_{\mathrm{e}}(t ; \omega)$ are respectively functionals of the observations $y_{\mathrm{p}}$ and $y_{\mathrm{e}}$ up to time $t$. 
Finally, we assume that both the players know and have perfect recall to the system characteristics $\Sigma$

$$
\Sigma \triangleq\left\{A, B_{\mathrm{p}}, B_{\mathrm{e}}, C_{\mathrm{p}}, C_{\mathrm{e}}, F, G_{\mathrm{p}}, G_{\mathrm{e}}, P_{0}, \bar{x}_{0}, Q_{\mathrm{f}}, Q, R_{\mathrm{p}}, R_{\mathrm{e}}\right\}
$$

and it is assumed that $x_{0}$ is independent of both the system and observation noises.

\section{Solution Method Involving Increasing Dimension}

In this and the following section, we propose two different techniques for solving the problem formulated above. The essential difficulty of this problem is that the pursuer does not know the observation of the evader and vice versa. The pursuer will have some knowledge about the evader's observation $y_{\mathrm{e}}$ through imperfect knowledge of the system state and he will try to use that knowledge lo estimate $y_{\mathrm{e}}$. The same holds for the evader.

The solution method of this section may be termed the method of alternate substitutions. First assume that $u_{\mathrm{e}} \equiv 0$. This is assumed to be known to the pursuer. The pursuer then faces a stochastic control problem which he can solve. The solution is denoted by $u_{\mathrm{p}}^{0}$. Then $u_{\mathrm{p}}^{0}$ is kept fixed, assumed to be known to the evader who then tries to maximize EJ w.r.t. $u_{\mathrm{e}}$. This yields a solution $u_{\mathrm{e}}^{0}$. Then again this solution is kept fixed, assumed known to the pursuer, who then solves his optimal control problem leading to $u_{\mathrm{p}}^{1}$, etc. If the sequence $u_{\mathrm{p}}^{0}, u_{\mathrm{p}}^{1}, \ldots$ and the sequence $u_{\mathrm{e}}^{0}, u_{\mathrm{e}}^{1}, \ldots$ converge, the limits will be called the solution to the problem. In mathematical terms, we get the following:

Step 1

Suppose $u_{\mathrm{e}} \equiv 0$. The pursuer faces the problem

$$
\begin{aligned}
& \dot{x}=A x+B_{\mathrm{p}} u_{\mathrm{p}}+F n, \\
& y_{\mathrm{p}}=C_{\mathrm{p}} x+G_{\mathrm{p}} n, \\
& \quad \min _{u_{\mathrm{p}}} \cdot \mathrm{E}\left\{\left\langle x(T), Q_{\mathrm{f}} x(T)\right\rangle+\int_{0}^{T}\left[\langle x, Q x\rangle+\left\langle u_{\mathrm{p}}, R_{\mathrm{p}} u_{\mathrm{p}}\right\rangle\right] \mathrm{d} t\right\}
\end{aligned}
$$

which is a standard problem whose solution is

$$
u_{\mathrm{p}}^{\mathrm{O}}(t)=K_{\mathrm{p}}^{\mathrm{O}}(t) \hat{z}_{\mathrm{p}}^{\mathrm{O}}(t)
$$

where $K_{\mathrm{p}}^{0}(t)$ is the control gain, satisfying a Riccati equation and

$$
\hat{z}_{\mathrm{p}}^{0}(t)=\mathrm{E}\left[x(t) \mid y_{\mathrm{p}}(s), \quad 0 \leqslant s \leqslant t ; u_{\mathrm{e}} \equiv 0\right] .
$$

The estimate $\hat{z}_{\mathrm{p}}^{0}(t)$ can be obtained by the standard Kalman filter technique; it satisfies a linear differential equation with $y_{\mathrm{p}}(\cdot)$ as the forcing term.

Step 2

Keep $u_{\mathrm{p}}^{0}(t)$ fixed and solve the maximization problem for the evader. Since the evader does not know $\hat{z}_{\mathrm{p}}^{\mathrm{o}}(t)$, the differential equation for $\hat{z}_{\mathrm{p}}^{\mathrm{O}}(t)$ is added to the original state equation and the new state $z_{\mathrm{e}}^{0^{\prime}} \triangleq\left(x^{\prime}, \hat{z}_{\mathrm{p}}^{0^{\prime}}\right)$ becomes a state of dimension $2 n$. The 
solution to the maximization problem can be written as

$$
\begin{aligned}
& u_{\mathrm{e}}^{0}(t)=K_{\mathrm{e}}^{\mathrm{o}}(t) \hat{z}_{\mathrm{e}}^{\mathrm{O}}(t), \\
& \hat{z}_{\mathrm{e}}^{\mathrm{O}}(t) \triangleq \mathrm{E}\left[z_{\mathrm{e}}^{\mathrm{O}}(t) \mid y_{\mathrm{e}}(s), \quad 0 \leqslant s \leqslant t ; u_{\mathrm{p}}^{\mathrm{O}}(\cdot)\right] .
\end{aligned}
$$

The estimate $\hat{z}_{\mathrm{e}}^{0}(t)$ again satisfies a linear differential equation of Kalman filtering.

Step 3

Keep $u_{\mathrm{e}}^{0}(t)$ fixed and solve the minimization problem for the pursuer. The state now becomes $3 n$ dimensional:

$$
z_{\mathbf{p}}^{1^{\prime}} \triangleq\left(x^{\prime}, \hat{z}_{\mathrm{e}}^{0^{\prime}}\right)
$$

and the solution can be written as

$$
\begin{aligned}
& u_{\mathrm{p}}^{1}(t)=K_{\mathrm{p}}^{1}(t) \hat{z}_{\mathbf{p}}^{1}(t), \\
& \hat{z}_{\mathbf{p}}^{1}(t) \triangleq \mathrm{E}\left[z_{\mathrm{p}}^{1}(t) \mid y_{\mathrm{p}}(s), 0 \leqslant s \leqslant t ; u_{\mathrm{e}}^{O}(\cdot)\right] .
\end{aligned}
$$

\section{Step 4}

$\operatorname{Keep} u_{\mathrm{p}}^{1}(t)$ fixed and solve the maximization problem for the evader. The state now becomes $4 n$ dimensional

$$
z_{\mathbf{e}}^{1^{\prime}} \triangleq\left(x^{\prime}, \hat{z}_{\mathbf{p}}^{1}\right)
$$

and the solution can be written as

$$
\begin{aligned}
& u_{\mathrm{e}}^{1}(t)=K_{\mathrm{e}}^{1}(t) \hat{z}_{\mathrm{e}}^{1}(t), \\
& \hat{z}_{\mathrm{e}}^{1}(t) \triangleq \mathrm{E}\left[z_{\mathrm{e}}^{1}(t) \mid y_{\mathrm{e}}(s), 0 \leqslant s \leqslant t ; u_{\mathrm{p}}^{1}(\cdot)\right] .
\end{aligned}
$$

We can carry on like this to get a sequence of strategies $\left\{u_{\mathrm{p}}^{(m)}(t)\right\}$ and $\left\{u_{\mathrm{e}}^{(m)}(t)\right\}$. The dimension increases with $m$. To the knowledge of the authors, this approach has not been explicitly carried out. In the next section we present an alternate approach for solving the problem.

\section{Solution Method using Infinite Dimensional State Space}

We assume that the strategies $u_{\mathrm{p}}(t ; \omega)$ and $u_{\mathrm{e}}(t ; \omega)$ are linear functionals of the observations $y_{\mathrm{p}}(\cdot)$ and $y_{\mathrm{e}}(\cdot)$, respectively, and also on $\bar{x}_{0}$ so that

$$
\begin{aligned}
& u_{\mathrm{p}}(t)=\int_{0}^{t} N_{\mathrm{p}}(t, \tau) y_{\mathrm{p}}(\tau) \mathrm{d} \tau+Z_{\mathrm{p}}(t) \bar{x}_{0}, \\
& u_{\mathrm{e}}(t)=\int_{0}^{t} N_{\mathrm{e}}(t, \tau) y_{\mathrm{e}}(\tau) \mathrm{d} \tau+Z_{\mathrm{e}}(t) \bar{x}_{0}
\end{aligned}
$$

where the kernels $N_{\mathrm{p}}(t, \tau)$ and $N_{\mathrm{e}}(t, \tau)$ are differentiable with respect to $\tau$ and the partial derivatives w.r.t. $\tau$ are square integrable on the triangle $0 \leqslant \tau \leqslant t \leqslant T$ and where $Z_{\mathrm{p}}(t)$ and $Z_{\mathrm{c}}(t)$ are square integrable on $[0, T]$. We have to determine $N_{\mathrm{p}, \mathrm{e}}(t, \tau)$ and $Z_{\mathrm{p} . \mathrm{e}}(t)$ for the optimum strategies $u_{\mathrm{p}, \mathrm{e}}(t)$.

To determine the optimum $N_{\mathrm{p}, \mathrm{e}}$ and $Z_{\mathrm{p}, \mathrm{e}}$, we have to define new state variables in 
an infinite dimensional Hilbert space. This has been done in detail in (2) (where it has been assumed that $\bar{x}_{0}=0$ and therefore, $Z_{\mathrm{p}, \mathrm{e}}$ have not been determined). The idea is that if (2) is substituted into (5) and if the resulting equation is subsequently substituted into (1) and (4) with $N_{\mathrm{p}}$ and $Z_{\mathrm{p}}$ assumed fixed, we obtain a control problem for the evader. The resulting control problem is, however, nonstandard and can be put into the standard form if a suitable new state is defined from the original one which is infinite dimensional. After the introduction of appropriate infinite dimensional state variables, we have a standard optimal control problem for the evader, and the optimal $u_{\mathrm{e}}$ turns out to have exactly the form (6). This gives us $N_{\mathrm{e}}$ and $Z_{\mathrm{e}}$ in terms of the given functions $N_{\mathrm{p}}$ and $Z_{\mathrm{p}}$. Reversing the roles of the players, we can write $N_{\mathrm{p}}$ and $Z_{\mathrm{p}}$ in terms of $N_{\mathrm{e}}$ and $Z_{\mathrm{e}}$. These give us four equations in four unknowns: $N_{\mathrm{p}, \mathrm{e}}$ and $Z_{\mathrm{p}, \mathrm{e}}$. They are given by

$$
\begin{array}{cl}
N_{\mathrm{p}}(t, \tau)=-R_{\mathrm{p}}^{-1}(t) B_{\mathrm{p}}(t)^{\prime}\left\{h_{\mathrm{p}}(T)+\int_{t}^{T} h_{\mathrm{p}}(s) \mathrm{d} s\right\} & 0 \leqslant \tau \leqslant t \leqslant T, \\
N_{\mathrm{e}}(t, \tau)=-R_{\mathrm{e}}^{-1}(t) B_{\mathrm{c}}(t)^{\prime}\left\{h_{\mathrm{c}}(T)+\int_{t}^{T} h_{\mathrm{c}}(s) \mathrm{d} s\right\} & 0 \leqslant \tau \leqslant t \leqslant T, \\
Z_{\mathrm{p}}(t)=-R_{\mathrm{p}}^{-1}(t) B_{\mathrm{p}}(t)^{\prime}\left\{g_{\mathrm{p}}(T)+\int_{t}^{T} g_{\mathrm{p}}(s) \mathrm{d} s\right\} & 0 \leqslant t \leqslant T, \\
Z_{\mathrm{e}}(t)=-R_{\mathrm{e}}^{-1}(t) B_{\mathrm{e}}(t)^{\prime}\left\{g_{\mathrm{e}}(T)+\int_{t}^{T} g_{\mathrm{e}}(s) \mathrm{d} s\right\} & 0 \leqslant t \leqslant T
\end{array}
$$

where

$$
\begin{aligned}
& h_{\mathrm{p}}(s)=\sum_{i=1}^{2}\left[\int_{0}^{T} K_{\mathrm{p} 1 i}(t, s, \sigma) S_{\mathrm{p} i}(t, \tau, \sigma) \mathrm{d} \sigma\right. \\
& \left.\quad+\underline{k}_{\mathrm{p} 1 i}(t, s) S_{\mathrm{p} i}(t, \tau, 0)+\bar{k}_{\mathrm{p} 1 i}(t, s) S_{\mathrm{p} i}(t, \tau, T)\right]
\end{aligned}
$$

and similar expressions hold for $h_{\mathrm{e}}(s)$ and $g_{\mathrm{e}}(s)$. The functions $K_{\mathrm{p} 1 i}, K_{\mathrm{e} 1 i}, S_{\mathrm{p} i}, S_{\mathrm{e} i}, k_{\mathrm{p} 1 i}$, $\bar{k}_{\mathrm{p} 1 i}, \underline{\mathrm{e}} 1 i_{1}, \overline{\mathrm{e}}_{\mathrm{e} 1 i}, i=1,2$, are described by a set of 34 coupled integro-partial differential equations with mixed boundary conditions. These equations have been explicitly given in (2) and will be used in the numerical procedure to be described in the next section. The equations for $\Phi_{\mathrm{p} 1 i}, i=1,2$, are given in (6) and are repeated in the Appendix for easy reference. The equations for $\Phi_{\mathrm{e} 1 i}, i=1,2$, can be obtained by interchanging the indices $\mathrm{p}$ and e. Note that Eq. (7) gives $N_{\mathrm{p}, \mathrm{e}}$ and $Z_{\mathrm{p}, \mathrm{e}}$ implicitly. If we write

$$
\mu=\left(R_{\mathrm{p}}^{-1}, R_{\mathrm{e}}^{-1}\right),
$$

it has been shown in (2) that (7) has a unique solution for $\|\mu\|$ sufficiently small. 


\section{Numerical Scheme}

We assume in what follows that $\bar{x}_{0}=0$ and therefore, we do not need to calculate $Z_{\mathrm{p}, \mathrm{e}}(t)$. We write Eq. (7) in a form more convenient for developing the numerical procedure to be described below. We rewrite Eq. (7) as

$$
z=\mathscr{H}(z, \mu)
$$

where $\mu$ is defined in (10) and where

$$
z^{\prime}=\left(z_{1}, z_{2}, z_{3}, z_{4}\right)=\left[R_{\mathrm{p}} \frac{\partial}{\partial \tau} N_{\mathrm{p}}(t, \tau), R_{\mathrm{e}} \frac{\partial}{\partial \tau} N_{\mathrm{e}}(t, \tau), N_{\mathrm{p}}(t, 0), N_{\mathrm{e}}(t, 0)\right] .
$$

This is explained in detail in (2). It is further shown in (2) that for $\|\mu\|$ sufficiently small, the sequence $\left\{z_{(i)}\right\}$ defined by

$$
z_{(i+1)}=\mathscr{H}\left(z_{(i)}, \mu\right), i=0,1,2, \ldots ; z_{(0)}=0
$$

converges and that the limit yields the desired solution to the differential game. We use a slightly different numerical scheme from the one described in (12). At each iteration, we use new values of the components of the vector $z$ already updated. More explicitly, suppose that we know $z_{(i)}=\left(z_{1(i)}, z_{2(i)}, z_{3_{(i)}}, z_{4(i)}\right)$. These values are substituted in (12) to calculate $z_{1(i+1)}$ and $z_{3(i+1)}$ (which, in turn, determines the new $\left.N_{\mathrm{p}}\right)$. Next, we use $z_{1(i+1)}, z_{2(i)}, z_{\mathbf{3}(i+1)}, z_{4(i)}$ in (12) to calculate $z_{2(i+1)}$ and $z_{4(i+1)}$ to obtain the updated $z_{(i+1)}$.

Each complete iteration, say, from $z_{(i)}$ to $z_{(i+1)}$ requires the solution of a set of 34 coupled integro-partial differential equations. Because of the size and complexity of these equations, a simple discretization scheme is used for their solution. We illustrate the scheme by means of one example. Consider the equation for $K_{\mathrm{p} 1 \text { i }}$, which can be written as

$$
\frac{\partial K_{\mathrm{p} 1 i}(t, s, \sigma)}{\partial t}=f(t, s, \sigma), K_{\mathrm{p} 1 i}(T, s, \sigma)=0,0 \leqslant s, \sigma \leqslant T
$$

where the explicit expression for $f$ may be found in (2). If $\Delta t=T / N$ where $N$ is the number of discretization steps, we write $t_{j}=s_{j}=\sigma_{j}=j \cdot \Delta t, j=0,1, \ldots, N$. We discretize Eq. (13) as

$$
\begin{gathered}
K_{\mathrm{p} 1 i}\left(t_{j-1}, s_{l}, \sigma_{m}\right)=K_{\mathrm{p} 1 i}\left(t_{j}, s_{l}, \sigma_{m}\right)-\Delta t \cdot f\left(t_{j}, s_{l}, \sigma_{m}\right), \\
K_{\mathrm{p} 1 i}\left(t_{N}, s_{l}, \sigma_{m}\right)=0 ; l, m=0,1, \ldots, N .
\end{gathered}
$$

In order to compute the integrals appearing in the expression for $f$, the integrals are replaced by stepfunctions with step size $\Delta t$. After each update, we calculate

$$
V=\sum_{j=0}^{N} \sum_{l=0}^{j}\left\{\left\|N_{\mathrm{p}}\left(t_{j}, \tau_{l}\right)_{(i+1)}-N_{\mathrm{p}}\left(t_{j}, \tau_{l}\right)_{(i)}\right\|^{2}+\left\|N_{\mathrm{e}}\left(t_{j}, \tau_{l}\right)_{(i+1)}-N_{\mathrm{e}}\left(t_{j}, \tau_{l}\right)_{(i)}\right\|^{2}\right\}
$$

where $\tau_{l}=l \cdot \Delta t$ and use $V<\varepsilon$ (a pre-assigned small quantity) as the stopping criterion. 


\section{Numerical Results}

For numerical simulation, we consider the following scalar example:

$$
\begin{aligned}
\dot{x}(t) & =u_{\mathrm{p}}(t)+u_{\mathrm{e}}(t)+F n(t) ; 0 \leqslant t \leqslant 1, \\
x(0) & =x_{0} ; x_{0} \sim N(0,0 \cdot 1), \\
y_{\mathrm{p}}(t) & =x(t)+G_{\mathrm{p}} n(t), \\
y_{\mathrm{e}}(t) & =x(t)+G_{\mathrm{e}} n(t), \\
J & =\frac{1}{2}\left\{a x^{2}(1)+\int_{0}^{1}\left[x^{2}(t)+u_{\mathrm{p}}^{2}(t)-5 u_{\mathrm{e}}^{2}(t)\right] \mathrm{d} t\right\} .
\end{aligned}
$$

A number of simulation runs were made with different values of the parameters given in Table I.

Examples 1 and 2 differ only in the terminal condition. Examples 2, 3 and 4 have increasing values of $G_{\mathrm{e}}$, while Examples 2,5 and 6 have increasing values of $G_{\mathrm{p}}$. Examples 1 and 7 have different $F$. Another example with $F=4$ with remaining parameters as in Example 7 showed divergence of our iteration scheme. The number of iterations to achieve the desired degree of accuracy was 3 in all cases except in Example 7 where the number of iterations needed was 5 (in spite of a larger value of $\varepsilon$ ).

In Table II, numerical values obtained for the function $N_{\mathrm{p}}$ are given, while in Table III, those for the function $N_{\mathrm{e}}$ are given. In all the examples we see that $\left|N_{\mathrm{p}, \mathrm{e}}(t, \tau)\right|$, for fixed $t$, are increasing functions of $\tau$ implying that recent observations are given greater weight than those obtained earlier, a very plausible fact. In Examples 2-6, we take the parameter $a$ to be zero. Due to our simple discretization scheme, this leads to $N_{\mathrm{p}}(T, \tau)=N_{\mathrm{e}}(T, \tau)=0$ for $0 \leqslant \tau \leqslant T=1$.

Comparing the various examples, we can draw a number of conclusions. In Examples 2, 3 and 4 , we see that with increasing $G_{e},\left|N_{\mathrm{e}}(t, \tau)\right|$ becomes smaller, indicating that if the measurement noise for the evader increases, he is going to rely less and less on his own observations. In Example 4, noise dominates observation so much that the evader hardly uses this information and thus his control resembles the open loop solution $\left(u_{\mathrm{e}}(t) \equiv 0\right)$ in which there are no measurements at all. We observe the same phenomenon for the pursuer in comparing Examples 2, 5 and 6 . In

TABLE I

\begin{tabular}{cllllll}
\hline Example & $F$ & $G_{\mathrm{p}}$ & $G_{\mathrm{e}}$ & $a$ & $\Delta t$ & $\varepsilon$ \\
1 & 0.4 & 0.4 & 0.4 & 1 & 0.2 & $10^{-4}$ \\
2 & 0.4 & 0.4 & 0.4 & 0 & 0.2 & $10^{-4}$ \\
3 & 0.4 & 0.4 & 1 & 0 & 0.2 & $10^{-4}$ \\
4 & 0.4 & 0.4 & 4 & 0 & 0.2 & $10^{-4}$ \\
5 & 0.4 & 1 & 0.4 & 0 & 0.2 & $10^{-4}$ \\
6 & 0.4 & 4 & 0.4 & 0 & 0.2 & $10^{-4}$ \\
7 & 1 & 0.4 & 0.4 & 1 & 0.2 & $10^{-2}$
\end{tabular}




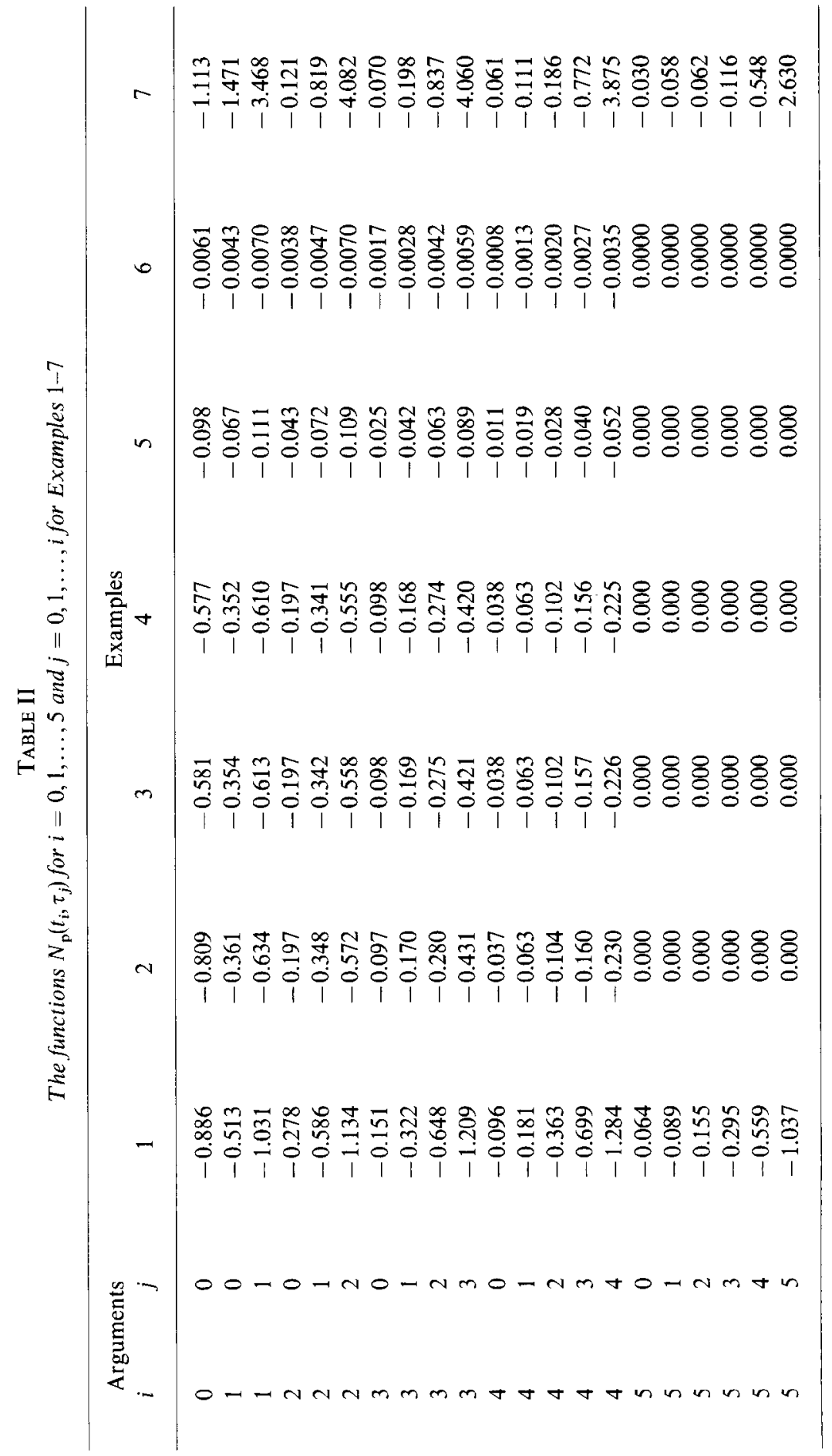




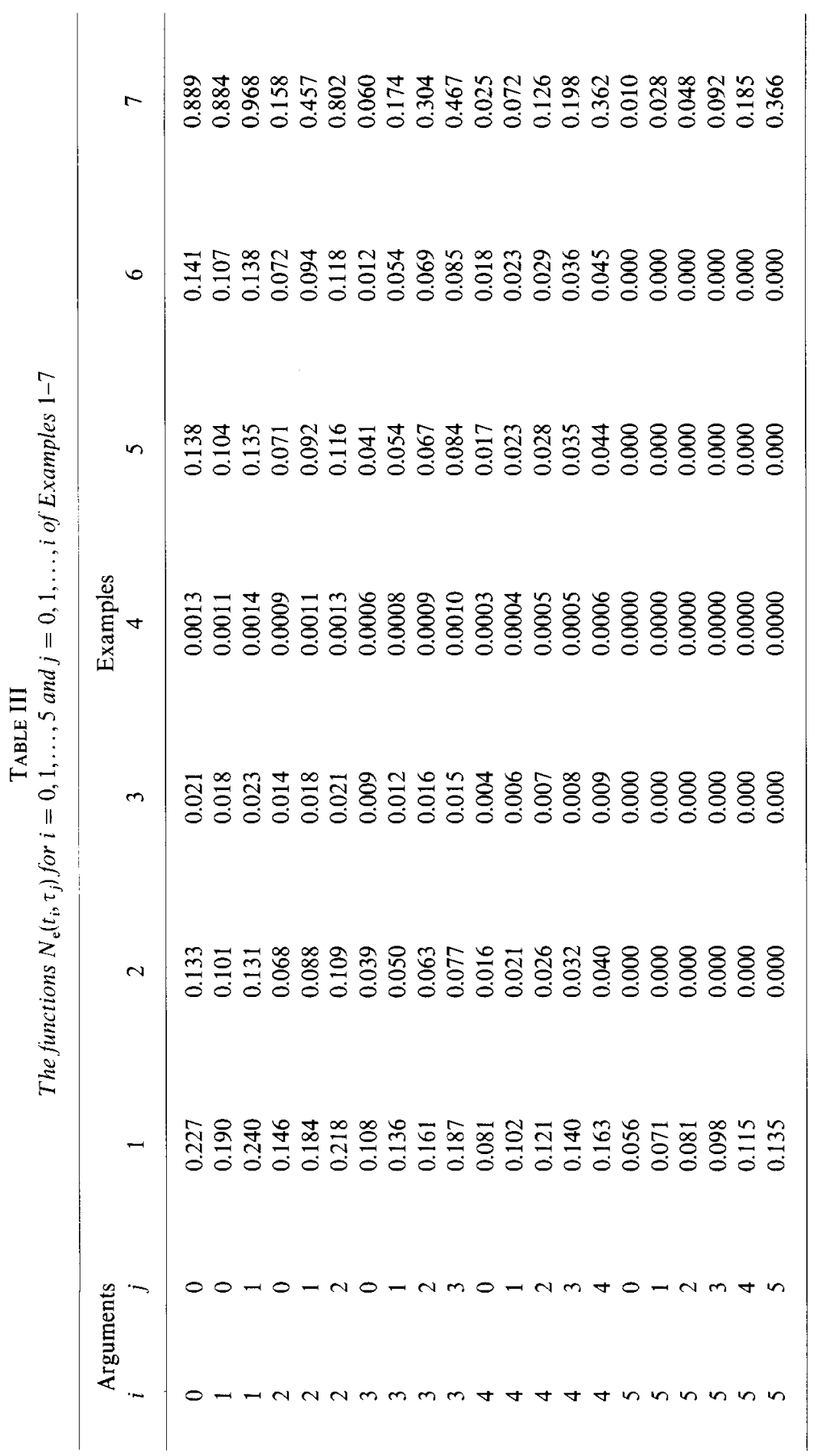


Example 7, system noise has been increased (w.r.t. that in Example 1), which leads to a slower convergence of the iteration scheme. Tables II and III also show that with an increasing $F$, the functions $\left|N_{\mathrm{p}}(t, \tau)\right|$ and $\left|N_{\mathrm{e}}(t, \tau)\right|$ also increase in value, as is to be expected. The execution time on a DEC-10 computer for each of the examples was in the order of $2 \mathrm{~s}$. A reduction in step size $(\Delta t=0.1)$ leads to the same conclusions.

\section{Conclusion}

A zero-sum linear quadratic Gaussian differential game has been considered in which the two players have possibly different noisy measurements. These different measurements lead to questions of the kind: "does the opponent know that I know that he...?" Two approaches to obtain the saddle-point solution have been discussed. The second approach has been worked out in detail by the authors in an earlier paper and the numerical results using that approach are presented here. In spite of the fact that the solution is given by a complicated set of integro-partial differential equations, the numerical results look plausible and promising. Use of more refined numerical scheme will undoubtedly lead to solutions closer to the theoretical ones.

\section{Acknowledyement}

The authors are indebted to Mr H. C. van Driel who did the numerical work as described in this paper.

\section{References}

(1) W. W. Wilman, "Formal solutions for a class of stochastic pursuit-evasion games," IEEE Trans. Aut. Control, Vol. AC-14, pp. 504-509, 1969.

(2) A. Bagchi and G. J. Olsder, "Linear-quadratic stochastic pursuit-evasion games," Appl. Math. Optimiz., Vol. 7, pp. 95-123, 1981.

(3) P. R. Kumar and J. H. van Schuppen, "On Nash equilibrium solutions in stochastic dynamical games," IEEE Trans. Aut. Control, Vol. AC-25, pp. 1146-1149, 1980.

(4) H. Kwakernaak and R. Sivan, "Linear Optimal Control Systems", John Wiley, New York, 1972.

(5) A. V. Balakrishnan, "Applied Functional Analysis", Springer, Berlin, 1976.

(6) H. van Driel, "Linear-quadratic differential games with stochastic observations", M.Sc. Thesis, Twente University of Technology, Enschede, The Netherlands, 1980 (in Dutch). 


\section{Appendix}

In this appendix, we give the integro-partial differential equation describing $\Phi_{\mathrm{p} 11}, \Phi_{\mathrm{p} 12}$ (see Eq. (9)). The operator $I_{s, t}$ is given by

$$
\begin{gathered}
I_{\mathrm{s}, t}=\left\{\begin{array}{l}
0 \text { for } s<t \\
I \text { for } s \geqslant t .
\end{array}\right. \\
\frac{\partial \Phi_{\mathrm{p} 11}(t, 0, s)}{\partial t}=I_{\mathrm{s}, \mathrm{t}}\left[A \Phi_{\mathrm{p} 11}(t, 0, T)+B_{\mathrm{e}}\left\{\int_{0}^{t} N_{\mathrm{e}}(t, \sigma) C_{\mathrm{e}}(\sigma) \Phi_{\mathrm{p} 11}(t, 0, \sigma) \mathrm{d} \sigma+\right.\right. \\
Z_{\mathrm{e}} \Phi_{\mathrm{p} 11}(t, 0,0)+N_{\mathrm{e}}(t, t) \Phi_{\mathrm{p} 21}(t, 0, T)-N_{\mathrm{e}}(t, 0) \Phi_{\mathrm{p} 21}(t, 0,0) \\
\left.\left.-\int_{0}^{t}\left(\frac{\partial}{\partial \rho} N_{\mathrm{e}}(t, \rho)\right) \Phi_{\mathrm{p} 21}(t, 0, \rho) \mathrm{d} \rho\right\}+B_{\mathrm{p}} Z_{\mathrm{p}}\right]-\left(\bar{p}_{11}(t, s) C_{\mathrm{p}}^{\prime}+I_{s, t} F G_{\mathrm{p}}^{\prime}\right)\left(G_{\mathrm{p}} G_{\mathrm{p}}^{\prime}\right)^{-1} C_{\mathrm{p}} \Phi_{\mathrm{p} 11}(t, 0, T), \\
\Phi_{\mathrm{p} 11}(0,0, s)-I, 0 \leqslant s \leqslant T, \\
\frac{\partial \Phi_{\mathrm{p} 21}(t, 0, s)}{\partial t}=-\left(\bar{p}_{21}(t, s) C_{\mathrm{p}}^{\prime}+I_{s, t} G_{\mathrm{e}} G_{\mathrm{p}}^{\prime}\right)\left(G_{\mathrm{p}} G_{\mathrm{p}}^{\prime}\right)^{-1} C_{\mathrm{p}} \Phi_{\mathrm{p} 11}(t, 0, T), \\
\Phi_{\mathrm{p} 211}(0,0, s)=0,0 \leqslant s \leqslant T .
\end{gathered}
$$

